\title{
Influencia de los comunicados de prensa de las publicaciones médicas sobre la calidad de la cobertura de los periódicos
}

\author{
Influence of medical journal press releases on the quality of associated newspaper coverage
}

Schwartz L y cols. BMJ 2012 Enero 27;344:d8164

\section{Objetivos}

Establecer la relación existente entre la calidad de los comunicados de prensa (CP) emitidos por las revistas médicas y la de las notas publicadas en periódicos (NP) internacionales redactadas por periodistas especializados.

\section{Diseño y población}

Fueron pesquisadas las primeras 100 investigaciones originales con resultados cuantificables y sus $68 \mathrm{CP}$ publicados durante 2009 en Annals of Internal Medicine, BMJ, Journal of the National Cancer Institute, JAMA y New England Journal of Medicine que generaron al menos una NP de más de 100 palabras en algún diario internacional. De las 759 NP, fue seleccionada una muestra aleatoria de 343 .
Evaluación de los predictores y medición de los resultados principales

Los investigadores establecieron criterios de calidad (mención del tamaño de la muestra y de los resultados, implicancia clínica, fuente de financiamiento de la investigación, incidencia de daños, etc.) de los 68 CP. Tomando las NP como unidad de análisis, calcularon la proporción de éstas que comunicaron dichos criterios de calidad (CC) y que no los comunicaron.

\section{Resultados principales}

Resultó más probable que una NP hubiera comunicado los CC si había sido escrita a partir de un CP que los había incluido, que si había sido escrita a partir de un CP que no los contenía $(p=0,0002)$. Ver los principales resultados en la tabla 1 .

Tabla 1: proporción de criterios de calidad presentes en las notas periodísticas de acuerdo a si el comunicado de prensa usado como insumo había reportado o no dicha información.

\begin{tabular}{|c|c|c|c|c|}
\hline \multicolumn{2}{|c|}{ Reportado por el comunicado de prensa } & $\begin{array}{l}\text { Reportado } \\
\text { por el comunicado } \\
\text { de prensa }\end{array}$ & $\begin{array}{l}\text { No reportado } \\
\text { por el comunicado } \\
\text { de prensa }\end{array}$ & $\begin{array}{l}\text { RR crudo } \\
\text { (IC95\%) }\end{array}$ \\
\hline \multicolumn{2}{|c|}{ Mención de la fuente de financiamiento } & $42 \%$ & $7 \%$ & $6,2(1,4$ a 27,0$)$ \\
\hline \multirow{2}{*}{ Resultado principal } & Cuantificación & $61 \%$ & $31 \%$ & $2,0(1,1$ a 3,7$)$ \\
\hline & Cuantificación con riesgos absolutos & $53 \%$ & $9 \%$ & $6,0(2,3$ a 15,4$)$ \\
\hline \multirow[t]{2}{*}{ Daños } & Mención & $68 \%$ & $24 \%$ & $2,8(1,1$ a 7,4$)$ \\
\hline & Cuantificación con riesgos absolutos & $43 \%$ & $3 \%$ & $14,1(1,9$ a 105$)$ \\
\hline \multicolumn{2}{|c|}{ Mención de limitaciones } & $48 \%$ & $17 \%$ & $3,0(1,5$ a 6,2$)$ \\
\hline
\end{tabular}

\section{Conclusión}

La calidad de los CP tiene un efecto directo sobre la calidad de las NP.

\section{Comentario}

Esta investigación documenta algo que los médicos solemos olvidar: la importancia de cómo se transmite la información, superando, en muchos casos incluso, la trascendencia de la información en sí. En el caso de los medios masivos de comunicación, esto cobra aún mayor significancia dado el gran impacto que éstos tienen sobre la percepción de la prevalencia y la gravedad de diferentes patologías, muchas veces exageradas intencionalmente y en el contexto de verdaderas campañas de promoción o tráfico de enfermedades ${ }^{1}$ (en inglés "disease mongering"), por ejemplo convirtiendo verdaderos factores de riesgo (como la osteoporosis ${ }^{2}$ ) o situaciones fisiológicas de la vida (la menopausia) en falsas condiciones percibidas como enfermedades con el objetivo de aumentar las ventas de alguna tecnología diagnóstica o terapéutica. Lamentablemente, y más allá de la segura existencia de los objetivos puramente comerciales como los expresados previamente, existe una barrera operativa y depen- diente del vértigo con el que trabajan los periodistas ${ }^{\ddagger} \mathrm{y}$ de la necesidad de conseguir noticias para sus publicaciones de alcance masivo. Por lo tanto, anunciar que se acaban de comunicar los resultados de una nueva droga para el tratamiento de alguna condición clínica "vende" más que discutir los problemas metodológicos que podría haber tenido la investigación en cuestión que sustenta dicho anuncio.

\section{Conclusiones de los comentadores}

Este artículo nos ayuda a comprender el proceso que contribuye a influenciar a la población y a los propios profesionales de la salud, y que es dependiente de la gran cantidad de instancias e interlocutores que existen desde el momento en que la información es publicada en una revista científica hasta que llega al público general.

Santiago Esteban [ Servicio de Medicina Familiar y Comunitaria, Hospital Italiano de Buenos Aires, santiago.esteban@hiba.org.ar ]

Sergio Terrasa [ Servicio de Medicina Familiar y Comunitaria, Hospital Italiano de Buenos Aires y Departamento de Salud Pública del Instituto Universitario Hospital Italiano sergio.terrasa@ hiba.org.ar ]

Esteban S y Terrasa S. Influencia de los comunicados de prensa de las publicaciones médicas sobre la calidad de la cobertura de los periódicos.Evid Act Práct Ambul. Jul-Set 2012;15(3):97. Comentado de: Schwartz L y cols. Influence of medical journal press releases on the quality of associated newspaper coverage: retrospective cohort study. BMJ. 2012 Jan 27;344:d8164. PMID 22286507.

\section{Referencias}

1. Vara A. Cómo medicalizar la vida diaria: la creación de enfermedades o "disease mongering". Evid. actual. práct. ambul; 11(5):130-132, Sept-Oct.2008. Disponible en URL: http://www.foroaps.org/files/ygjytgkgyt.pdf (último acceso 01/05/12).

2. Musso C y col. Anormalidad, inadaptación, enfermedad y salud: Los pilares de la interpretración clínica. Evid. actual. páct. ambul.9(2);50-51.Mar-Abr 2006.Disponible en URL: http://www.foroaps.org/files/pilares\%20de\%20la\%20interp.pdf (último acceso 01/05/12).

‡ A la mayoría de los periodistas responsables de las secciones "salud" de los diarios de circulación masiva les llega con una semana de anticipación los CP de las principales revistas médicas. 